\title{
Characterization of Magnetic Materials by Means of Neutron Scattering and Future Possibilities at a Next Generation Spallation Neutron Source
}

\author{
F. Klose* and G. Ehlers**
}

* Oak Ridge National Laboratory, Spallation Neutron Source Project, current address: Argonne National Laboratory, IPNS/SNS, 9700 South Cass Avenue, Argonne, IL 60439, USA ** Institute Laue-Langevin, 6, rue Jules Horowitz, BP 156, 38042 Grenoble Cedex 9, France

Due to its unique elementary characteristics, the neutron is especially suited for probing the magnetic properties of materials. It is not electrically charged, and therefore penetrates deeply into condensed matter. On the other hand, it possesses spin $1 / 2$, and so interacts with atoms as well as with magnetic moments present in matt er. These two types of interaction are of comparable strength. Since the neutron has mass, its energy is inversely proportional to the square of its de Broglie wavelength. Neutrons with wavelengths in the Angstroem range possess kinetic energies in the meV range, which are the typical energies of elementary excitations in condensed matter. Consequently, neutrons can simultaneously probe structural and magnetic spatial correlations on atomic to mesoscopic length scales, as well as structural and magnetic temporal correlations in the range of $10^{-}$ ${ }^{14}$ to $10^{-7} \mathrm{~s}$. By keeping track of the neutron spin and its change during the interaction with the sample, one can unambiguously separate nuclear and magnetic scattering processes.

Compared to other experimental techniques developed to investigate magnetic properties of matter, neutron scattering has particular merits: 1) transparent and easy experimental procedures that allow a straightforward conversion of experimental data into physical quantities, 2) a non -destructive nature, 3) sensitivity to both volume and surface properties, and 4) the capability to study a huge variety of magnetic phenomena and different classes of materials. The dependence of magnetic properties on temperature, pressure, or magnetic field can easily be explored since most sample environments can be made transparent to neutrons.

The applicability of neutron scattering techniques, however, is limited by the relatively low flux of useful neutrons generated by today's research reactors or pulsed $\mathrm{sp}$ allation sources, which is many orders of magnitude smaller than the flux of X -rays produced by contemporary photon sources. Recently, major efforts have been made to optimize existing sources and to develop new, more powerful sources.

A next -generation pulsed source, the Spallation Neutron Source (SNS), which has been under construction at Oak Ridge National Laboratory since 1999, will become operational in 2006. This facility will generate an effective neutron flux about one order of magnitude higher th an today's best neutron sources. Other approaches to gain intensity concern optimization of neutron optical components, development of new optical devices, and implementation of advanced instrument designs. Simulation calculations indicate that these approaches should further increase the usable flux by another order of magnitude for most SNS scattering instruments. The total intensity gain for SNS instruments, therefore, can be as high as two orders of magnitude, an improvement that will greatly enhance the quality of neutron scattering studies. 
Figure 1 shows the current layout of the neutron scattering instruments in the SNS target hall [1]. Currently, seven instruments have been approved for construction (a backscattering spectrometer, a magnetism reflectometer, a liquids reflectometer, a small -angle scattering instrument, a powder diffractometer, a wide angle chopper spectrometer and a cold neutron chopper spectrometer). Seven more instruments are in the design phase (a high -pressure diffractometer, an engineering diffractometer, a single -crystal diffractometer, a disordered materials diffractometer, a high resolution chopper spectrometer, a spin -echo spectrometer and a fundamental physics beamline). Construction will start as soon as funds become available. Further instruments will follow until eventually all 24 available SNS beam ports are occupied [2].

\section{References}

[1] For detailed information on particular instruments consult the SNS Instrument Systems web site at'wWW.sns.anl.gov,

[2] This work is supported by the Spallation Neutron Source Project (SNS). SNS is managed by UT-Battelle, LLC, under contract No. DE-AC05-000R22725 for the U.S. Department of Energy.

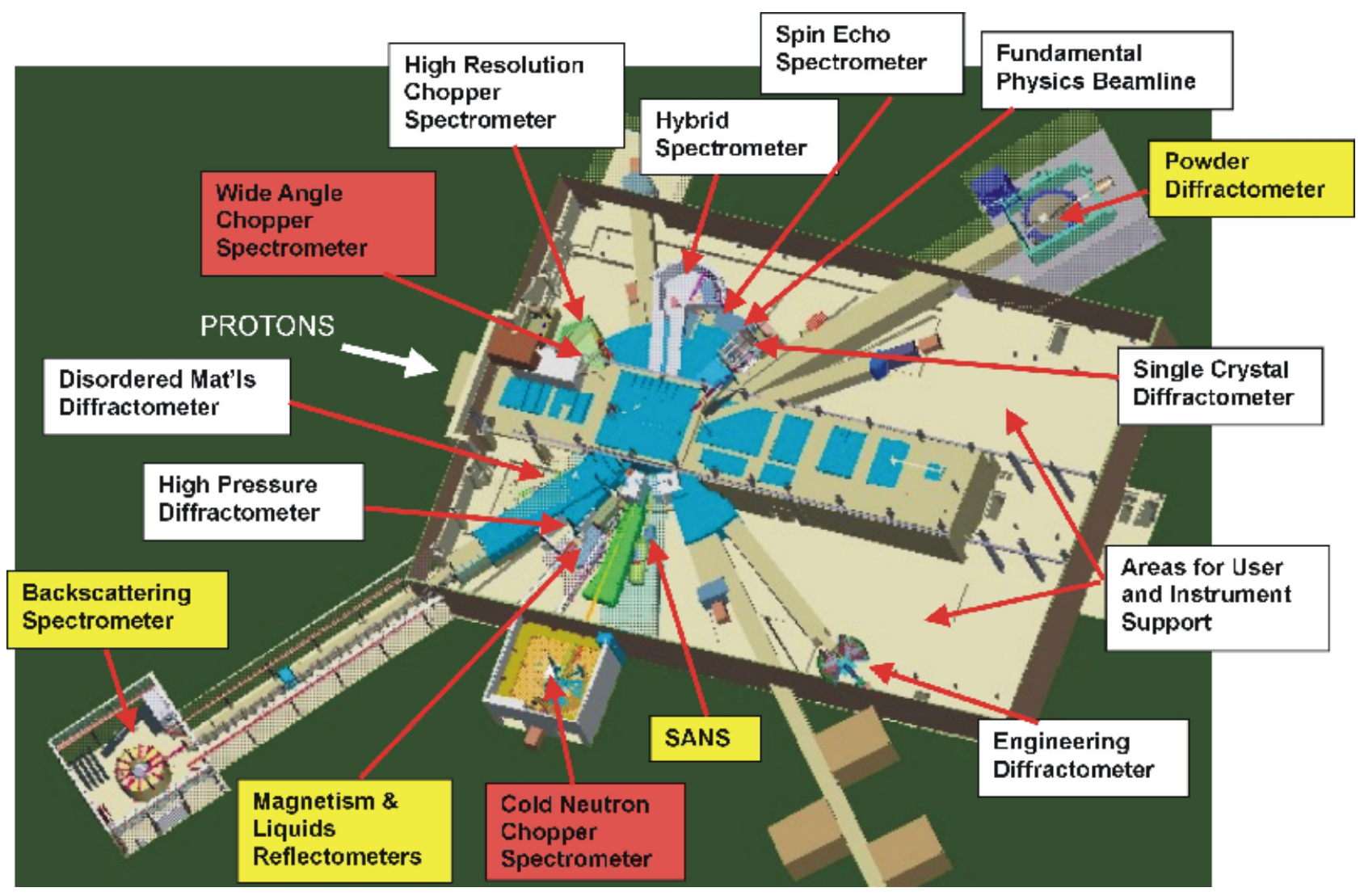

FIG. 1. Current layout of SNS target hall (size: $60 \mathrm{~m}$ x $90 \mathrm{~m}$ ) and neutron scattering instruments. The yellow background characterizes instruments that are funded by the SNS Project, red background instruments are funded by (university) instrument development teams and white background characterizes instruments that are currently in the design phase. 